\title{
Towards the Development of a Wireless Network Node Lifetime Calculation Tool
}

\author{
Waqas Ikram \\ Imperial College London \\ Department of Chemical Engineering, \\ London SW7 2AZ \\ waqas.ikram08@imperial.ac.uk
}

\begin{abstract}
The designers, optimizers and maintenance personnel of a wireless sensor network are frequently challenged by system level energy budget considerations. Minimizing the need for battery replacement is often the design goal while ensuring that a balance is maintained between capability and current consumption in order to address application needs. In this paper, a tool is introduced which can be used to calculate the lifetime of a battery operated wireless node. It allows the user to configure different wireless sensor platforms, select a battery of choice, and specify the application which needs to be executed over the configured hardware. As a result, the tool computes an estimate for the expected lifetime of the wireless sensor node. Furthermore, the tool also provides a detailed overview of the energy consumed by each component during a duty cycle.
\end{abstract}

\section{Introduction}

An omnipresent topic in Wireless Sensor Network (WSN) research is energy-efficiency, which leads to extension in battery life, and is crucial to achieve the required network lifetime. This theme permeates all levels of network design, ranging from battery selection and hardware design to the selection of communication protocols. A building block in a WSN is a wireless sensor node, a.k.a mote, and is expected to run unattended for several years without battery replacement. In a typical WSN application, such as, environmental monitoring, tens or hundreds of these nodes are expected to be scattered over a wider geographical area. Therefore, battery replacement in such a scenario is costly, time consuming, difficult and a cumbersome task. Consequently, the WSN applications are delimited due to the finite onboard energy available at the disposal of a wireless sensor node as it corresponds to network lifetime.

Despite the importance of battery lifetime, there are very few tools available for network node lifetime

\author{
Nina F. Thornhill \\ Imperial College London \\ Department of Chemical Engineering, \\ London SW7 2AZ \\ n.thornhill@imperial.ac.uk
}

estimation. On one hand, if they are available, typically they are in the form of an Excel spreadsheet, as in [1]. Mostly, they are configured to demonstrate the lifetime of a specific hardware node from a particular vendor, or the energy savings achievable using a particular communication protocol. On the other hand, MATSNL [2] is an example package comprising of MATLAB files for computing wireless sensor node lifetime. However, the topic of network node lifetime estimation requires a broader view, and encompasses various themes, including: battery, transceiver, memory, processing and sensing technology, in addition to the energy-efficient MAC and routing protocols. It can be appreciated that this topic can become quite complicated since it involves various disciplines. Moreover, the characteristics of these technologies vary with time, environment and usage. Furthermore, the use of mesh networks, random access protocols, and event-triggered applications can make the network node lifetime estimation process more sophisticated.

In order to bridge the gap between in-situ practice and theory, the overall designed solution acknowledges the limitation of reported literature, such as, on discharge curve of batteries during deployments. It can lead to battery model mismatch. According to [3], the reason is the time it takes to completely drain the battery. However, [4] highlights that effective battery capacity varies from vendor to vendor and that the peak current drain negatively impacts battery capacity. The tool presented here highlights and considers such issues in the overall proposed system design.

The layout of this work in progress paper is as follows. Section 2 highlights the building block of a WSN, i.e. a wireless sensor node and the batteries which power those nodes. Furthermore, the factors which are important to be considered in lifetime estimation are given in Section 3. In addition, Section 4 illustrates the model adopted for lifetime calculation. Thereafter, in Section 5 the outlook of the designed GUI is presented, followed by summary in Section 6 . 


\section{Building Block of a WSN}

In this section, a wireless sensor node is briefly introduced. A wireless node may even incorporate an actuator. However, within this paper the discussion is restricted to wireless sensors only. Furthermore, as these nodes are often battery operated, therefore a brief overview of batteries is provided as they influence a node's operational lifetime.

\subsection{Wireless Sensor Node}

A typical WSN incorporates the following: built-in radio transceiver, processing unit, peripherals (such as ADC), memory and sensor(s). Within a wireless network, each of these devices can support multiple sensors which can retrieve some physical characteristic from the environment. The sensed data is then dispatched to one or more sink nodes where it is collected, analyzed, and decisions are made.

\subsection{Overview of Batteries}

Batteries are energy storage devices which are rated by their capacity given in current-time units, milliampere-hours (mAh). It is a measure of stored charge contained within a battery under set conditions. When it is measured under set conditions it is referred to as rated capacity. The capacity of a battery is given by Eq. 1 [5]

$$
C=\int_{t_{i}}^{t_{f}} I(t) d t=\frac{1}{R} \int_{t_{i}}^{t_{f}} U(t) d t \quad \text { Eq. } 1
$$

where, $I(t)$ represents the value of current flowing out of the battery at time instance $t, U(t)$ represents voltage and $R$ represents resistance of the application. Therefore, it is seen that the capacity depends on supplied voltage level. Eq. 1 is often simplified to Eq. 2 [5]. It signifies that in a continuous drain application, the average current flowing multiplied by the hours of service, $t_{h}$, is the rated capacity of the battery.

$$
C=t_{h} I=t_{h} \times U / R \quad \text { Eq. } 2
$$

In an ideal battery, the relationship between drain current and discharge time is considered stable and absolute. However, in practice, it is not always true as it depends on factors such as battery chemistry, storage life, voltage, operating temperature and drain current [6]. Normally, a battery capacity is better at lower drain currents, or when operated near the specified test conditions $[5,6]$.

\section{Factors Affecting Lifetime Calculation}

\subsection{Battery Model}

The work presented by [7] reviews some of the circuit based battery models available in literature for simulation of battery behaviors. Amongst those presented models is a simple battery model, and is represented by an open circuit voltage, $E_{0}$, internal battery resistance, $R_{i n t}$, and terminal voltage $V_{0}$ of the battery. This model does not take into consideration the varying characteristics of internal resistance, such as its dependence on state of charge. Furthermore, the internal resistance is temperature sensitive. Moreover, other models have been proposed in literature, such as a model presented by [8], where the variations in internal resistance are taken into consideration.

In addition, other extensions to the simple battery model are available which take into consideration the factors such as, overvoltage resistance, capacitance, self-discharge, environmental temperature, electrolyte temperature and discharge current modes. The selection of the most relevant model depends on merits of selection of such a model. For instance, the Resistive Thevenin battery model assumes that the battery discharge occurs at a constant rate [7]. Such an assumption may not hold for systems with very low duty cycles, such as wireless sensor nodes. Furthermore, [9] presented a model for lifetime calculation of a battery considering both constant and variable loads.

\subsection{Time and Environmental Factors}

The commonly found and widely used batteries are: Lithium Thionyl Chloride, Alkaline-Manganese Dioxide and Nickel Metal Hydride batteries. An important observation from the discharge curves as found in $[10,11,12]$ reveals that the systems which are powered from Alkaline-Manganese Dioxide and Nickel Metal Hydride batteries need to tolerate a wide range of changes to supplied voltage. The cut-off voltage of an alkaline battery is $0.8 \mathrm{~V}$, which signifies that the manufacturer does not consider a battery as empty until its output falls to $0.8 \mathrm{~V}$. Furthermore, the Lithium batteries have an output which is steady for longer duration of service hours. This signifies that if a wireless node is operated using Lithium battery, it does not need to adjust to voltage changes as often, as is the case of Alkaline and Nickel based batteries. Also, the simplified battery capacity formula given in Eq. 2 is justified in the case of Lithium batteries.

The use of DC-to-DC converters can address the issue of varying battery voltage. However, their use is disappearing in new sensor hardware platform design [3]. In addition, lifetimes beyond certain years cannot be achieved because of battery self-discharge. Long life batteries such as Alkaline Manganese Dioxide batteries have shelf life of 2-3 years if stored at $21{ }^{\circ} \mathrm{C}$, and a shelf life of 2 months if stored at $54{ }^{\circ} \mathrm{C}$ [10]. The shelf life in this case is defined as the time after which a battery will still deliver $75 \%$ of its original specified capacity [13]. 


\subsection{Others}

- Current draw: In [4] it was noted that in some cases the achievable battery capacity was $50 \%$ of the maximum rated capacity, and that the peak current draw reduces effective battery capacity.

- Device Type: If a node performs additional tasks, such as routing in a mesh network then the duty cycle increases and the battery depletes quickly.

- Network protocol: Depending on the protocol of choice those protocols which use CSMA based Medium Access Control (MAC) layers introduce a random back-off variable in lifetime estimation. However, in the work presented here only TDMA based MACs are considered.

- Application communication rate: How often a node communicates either to communicate data or network health messages affect node lifetime. The more frequent the communication, the shorter the battery lifetime of the node.

- Transmission power control: It is a mechanism adopted to prolong node lifetime and minimize network interference. If adopted, then the current draw at the time of transmission will be a variable value and not a fixed current draw, as is the case without transmission power control.

- Recovery effect: For intermittent discharge, if the battery is left idling for sufficient amount of time then it can recover lost capacity [14].

\section{System Model}

An example is used to explain the implemented system design. A typical wireless sensor node performs the following tasks:

- Periodic sensing of the phenomena of interest.

- Transmission of the sensed data to the sink node.

- Waiting for an acknowledgment, if required.

- Retransmitting the data packet if needed.

- Listening to any incoming message if needed.

- Sleeping for the rest of the time up to the point of next scheduled periodic sensing.

Variants of these tasks will exist and depend on the application profile specified by the user. To summarise, the user specifies a duty-cycle through different tasks which are to be performed and for how long within a cycle. The kind of task which a device can undertake depends on its hardware capability, such as the peripherals it supports. Each of these tasks will require power to complete the designated task.

\subsection{Implemented System Model}

The overall system can be modelled as a switched resistance network, similar to the approach taken in [4], as shown in Figure 1. On the left of the figure is an electrical equivalent of a battery model. To begin with, a simple battery model is used. Later on new models can be incorporated. In parallel to the battery circuit, a capacitor or a voltage conditioning circuit (like a regulator) can be used. This region is highlighted in grey, and is left empty at this stage in time. On the right is the electrical equivalent of the system which is configured by the user. Each component is represented by its own individual parallel resistance block. Here, each resistor represents the current draw of a specific mode of a component in the designed system. The amount of time a node spends in a particular mode is represented by the duration of the switch status. The status of the switch is governed by the registers which are programmable. The reason for having multiple resistors in one block is to illustrate the different current consumption modes which are supported by the component on a device. Finally, the switch has to toggle between these available loads.

\subsection{Flow Chart}

A flow chart of the process involved in calculating the network node lifetime is depicted in Figure 2. The process starts by taking into consideration three inputs from the user which involves the design of the wireless node, selection of battery and specification of the application profile. From this information, states are defined. A state is represented here to show a particular task which a device indulges in, such as temperature sensing. Based on that information, the components which are required to conduct that task are configured and a switched resistor network is formulated as shown in Figure 1. Moreover, the time which each specific task takes is taken into consideration, which also involves the time duration related to communication protocol overheads, such as those linked to CSMA or TDMA based MAC protocols. From then onwards, a state table is formulated, which has three columns. They are state name, current drain and time duration.

\section{Designed GUI}

Figure 3 shows a snapshot of the designed Graphical User Interface (GUI) which is written in Csharp. There are many configurable features available, which are split into three control panels. The first control panel starting from the left is the panel which can be used to build the wireless sensor node hardware or use an existing commercially available mote. Selection of the power unit, i.e. the batteries, is also part of hardware specification. The next step is about defining the application profile which is used to build

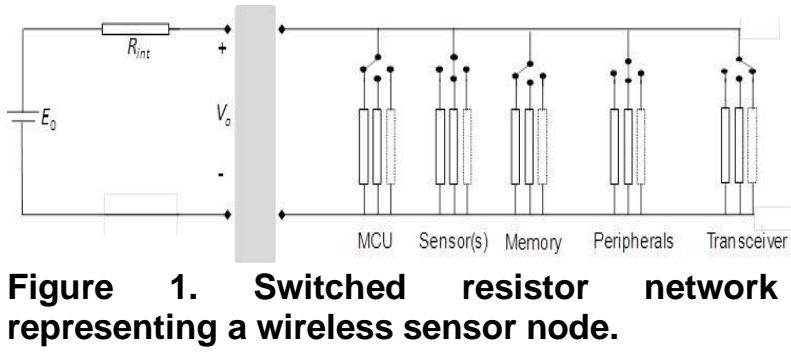




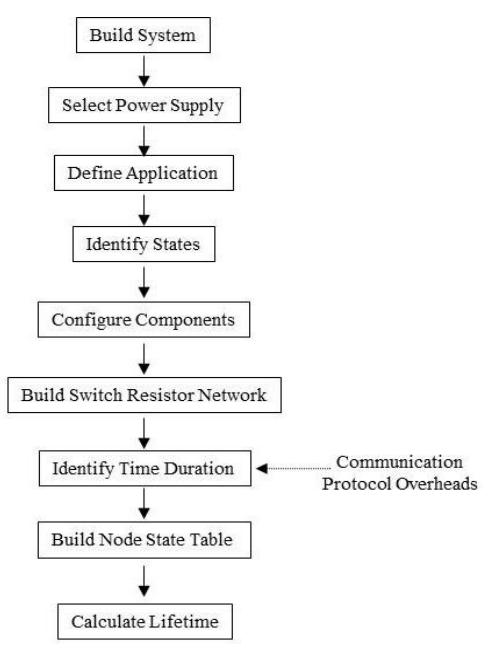

Figure 2. Flow chart of network node lifetime calculation.

the state table shown in Table 1. Finally, the tool then computes the lifetime estimation using the panel on the right. The outcome of this process is a value corresponding to expected node lifetime and a graphical illustration of the energy consumed per cycle.

\section{Summary and Future Work}

This WiP paper has presented a GUI designed for calculating the lifetime of a sensor node for a user specified application profile. The tool provides the user with the freedom to choose a sensor node platform from the available options provided in the tool or custom-build their own hardware node platform. After the selection of the wireless sensor node platform and

Table 1. Example representation of user defined states and associated current drain.

\begin{tabular}{|l|c|l|}
\hline \multicolumn{1}{|c|}{ STATE } & CURRENT DRAIN (mA) & DURATION (ms) \\
\hline Sensing & & \\
\hline Processing & \\
\hline Storing & \\
\hline Transmitting & & \\
\hline Receiving & & \\
\hline $\begin{array}{l}\text { Sleeping/ } \\
\text { Hibernation }\end{array}$ & & \\
\hline
\end{tabular}

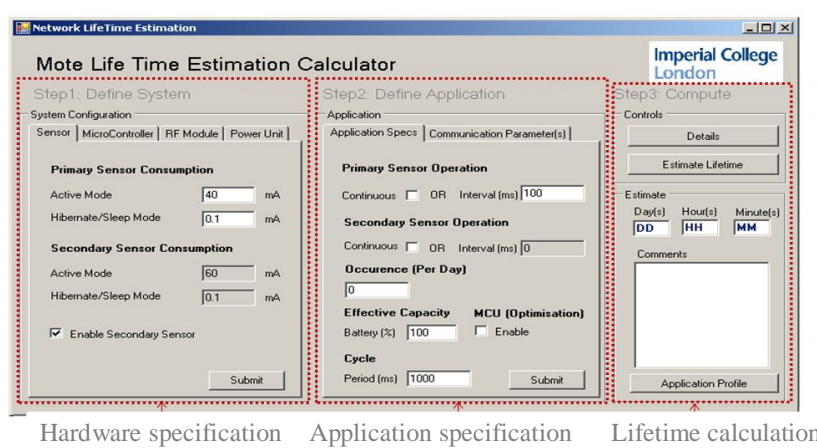

Figure 3. The GUI designed for network node lifetime calculation. the specification of the application profile, the tool allows the user to compute the node's lifetime against batteries with varying rated and delivered capacity. In this particular designed GUI, a TDMA based MAC protocol is assumed with periodic sensing.

In this initiative, various justified assumptions are made regarding the modeling of the system. However, new models can be built and used with this tool based on MS .NET. Shortly, this tool will be available at: http://www3.imperial.ac.uk/processautomation/research/wireless

\section{Acknowledgments}

The authors acknowledge the DTA Scholarship from Imperial College and funding from the ABB/RAEng Chair of Process Automation. The authors also acknowledge the contributions from anonymous reviewers for their detailed comments and suggestions to the manuscript.

\section{References}

[1] Crossbow, MICA2: Mote Battery Life Calculator, retrieved from: www.xbow.com, on (May. 6, 2011)

[2] MATSNL, MATLAB Wireless Sensor Node Platform Lifetime Prediction \& Simulation, http://web.adsc.com.sg/deokwoo.jung/research/MATSNL/ MATSNL.htm (Feb. 12, 2013).

[3] Nguyen,H.A., Forster, A.,Puccinelli, D., Sensor node lifetime: An experimental study, IEEE Conference on Pervasive Computing and Communications, USA, (Mar 21-25, 2011).

[4] Jensen, M., Coin cells and peak current draw, www.ti.com/lit/wp/swra349/swra349.pdf (Nov.12, '11).

[5] BIS, Establishing harmonized methods to determine the capacity of all portable and automotive batteries and rules for the use of label indicating the capacity of these batteries, from http://ec.europa.eu (May.4, 2011).

[6] Hill, J. L., System Architecture for Wireless Sensor Networks, PhD Thesis, University of California, Berkley, USA, 2003.

[7] González-Longatt, F. M., Circuit Based Battery Models: A Review, Congreso Iberoamericano de Estudiantes de Ingeniería Eléctrica, Puerto La Cruz, Venezuela (3-7th April, 2006).

[8] Cun, J. P., The Experience of a UPS company in advanced battery monitoring, MGE UPS Systems, Grenoble, retrieved from: http://www-merlingerin.eunet.fr/news/techpap/tp02us.ht (Jun. 12, 2012).

[9] Harper,A.C and Buress,R.V, Mobile Telephones: Networks, Applications, and Performance, Nova, New York, 2008.

[10] Duracell, Alkaline-manganese dioxide, http://www.duracell.com/media/enUS/pdf/gtcl/Technical_Bulletins/Alkaline\%20Technical\% 20Bulletin.pdf, (Jan. 12, 2011)

[11] Duracell, Nickel metal hydride battery, http://www.duracell.com/en-US/product/rechargeablebatteries.jspx (Jan. 12, 2011).

[12] Tadiran, iXtra- long term performance: Li/SOCl2 batteries, www.tadiranbatteries.de (Apr. 10, 2012).

[13] Crompton, T. R., Battery reference book, Newnes, London, 2000.

[14] Chau, C. K. at al.,Battery recovery aware sensor networks, Conference on Modeling and Optimization in Mobile, Ad Hoc, and Wireless Networks, Korea, (Jun. 23-25, '09). 